\title{
Dynamical and spectroscopic studies of nonrigid molecules. Application to the visible spectrum of thioacetaldehyde
}

Cite as: J. Chem. Phys. 93, 5786 (1990); https://doi.org/10.1063/1.459574

Submitted: 12 July 1989 . Accepted: 28 June 1990 . Published Online: 04 June 1998

Y. G. Smeyers, A. Niño, and D. C. Moule

\section{ARTICLES YOU MAY BE INTERESTED IN}

Thiocarbonyl spectroscopy: Methyl torsional vibrations and internal rotational barriers of thioacetaldehyde in its $\widetilde{a}^{3} \mathrm{~A}^{\prime}$ and $\mathrm{X}^{1} \mathrm{~A}^{\prime}$ states

The Journal of Chemical Physics 87, 60 (1987); https://doi.org/10.1063/1.453554

General Internal Motion of Molecules, Classical and Quantum-Mechanical Hamiltonian The Journal of Chemical Physics 49, 1510 (1968); https://doi.org/10.1063/1.1670272

Vibration-Rotation Interactions and the Choice of Rotating Axes for Polyatomic Molecules The Journal of Chemical Physics 56, 1715 (1972); https://doi.org/10.1063/1.1677430

\section{Lock-in Amplifiers up to $600 \mathrm{MHz}$}
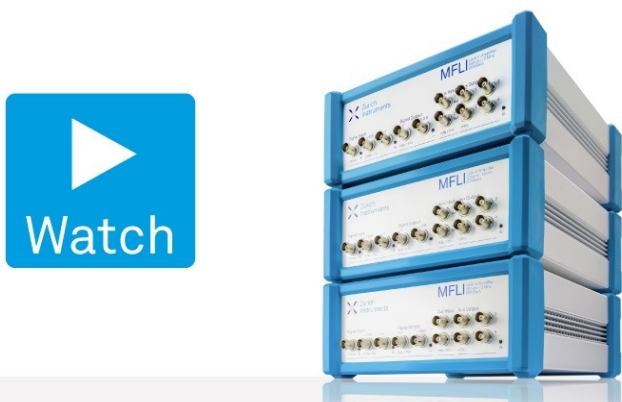


\title{
Dynamical and spectroscopic studies of nonrigid molecules. Application to the visible spectrum of thioacetaldehyde
}

\author{
Y. G. Smeyers and A. Niño \\ Instituto de Estructura de la Materia, CSIC, c/Serrano, 119, 28006 Madrid, Spain \\ D. C. Moule \\ Department of Chemistry, Brock University, St. Catharines, Ontario L2S3A1, Canada
}

(Received 12 July 1989; accepted 28 June 1990)

\begin{abstract}
The methyl torsion and aldehydic hydrogen wagging modes are studied theoretically in thioacetaldehyde, in both ground and first triplet excited states. For this purpose, the potential energy surfaces were determined by $a b$ initio restricted Hartree Fock and unrestricted Hartree Fock calculations with $4-31$ basis set $+d$ orbitals on the sulphur atom. The two electronic states were found to have different preferred conformations. The singlet state exhibits a planar eclipsed conformation, whereas the structure of the triplet state is anti-eclipsed and pyramidal. It was found that a potential function, which was constructed from a symmetry adapted double Fourier expansion of the wagging and torsional coordinates, gave a reasonable fit to the energy points. The two-dimensional Schrödinger equations for the torsion and wagging motions were solved for both singlet and triplet states, taking into account the internal symmetry and the appropriate basis. The relative band locations and the intensities (Franck-Condon factors) were evaluated from the eigenvalues and the eigenvectors. Spectra were calculated for the thioacetaldehyde $\mathrm{CH}_{3} \mathrm{CHS} / \mathrm{CH}_{3} \mathrm{CDS} / \mathrm{CD}_{3} \mathrm{CHS} / \mathrm{CD}_{3} \mathrm{CDS}$ isotopomers. New assignments for the higher bands in the $\mathrm{CH}_{3} \mathrm{CHS}$ spectrum are proposed.
\end{abstract}

\section{INTRODUCTION}

The properties of the electronic ground states $\widetilde{X}\left(S_{0}\right)$ of medium-sized molecules containing the carbonyl moiety have been extensively studied by microwave, infrared, and Raman spectroscopies. It has only been in the last few years $^{1,2}$ that the vibrational and rotational dynamics of the higher electronic $\widetilde{a}, \widetilde{A}, \widetilde{B}, \ldots$ states have been experimentally determined through a combination of supersonic-jet-laserexcitation and classical spectroscopies. The assignments and the analyses of the more complex systems have been assisted by quantum mechanical $a b$ initio self-consistent field (SCF) calculations that have led to a better understanding of the structure and the dynamics of the higher electronic states. ${ }^{3,4}$

The parallel development in the study ${ }^{5}$ of the thiocarbonyl group of compounds has been hampered by two factors. Most of the aliphatic thiocarbonyl aldehydes are unstable in the vapor phase at low pressures, moreover they possess a vile smell. Thioacetaldehyde, $\mathrm{CH}_{3} \mathrm{CHS}$, is unstable in the vapor phase with a half-life of about $10 \mathrm{~s}$. It can be prepared from the trimer 1,3,5-trimethyl-s-trithane by flash pyrolysis. In all, six electronic systems have been observed $^{6}$ in absorption. The spectrum ${ }^{7}$ that lies in the visible, $490-620 \mathrm{~nm}$, can be recorded in absorption at path lengths of $60 \mathrm{~m}$. It is found to consist of two systems of nearly equal intensity that have been attributed to $n \rightarrow \pi^{*}$ excitation. These have been assigned to the spin allowed $S_{1} \leftarrow S_{0}, \quad \widetilde{A}^{1} A^{\prime \prime} \leftarrow \widetilde{X}^{1} A^{\prime}$ and spin forbidden $T_{1} \leftarrow S_{0}$, $\widetilde{a}^{3} A^{\prime \prime}-\widetilde{X}^{1} A^{\prime}$ electronic transitions. Both systems are complex and congested. The lower $T_{1}$ state was observed to be radiative ${ }^{8}$ with a lifetime under collision-free conditions of $10 \mu \mathrm{s}$. Weak emission was detected following excitation to the $S_{1}$ state. It is believed to result from collisionally assisted intersystem crossing to the $T_{1}$ state.

Because of the more restrictive nature of the vibronic spin orbit selection rules that operate for triplet-singlet transitions, the $T_{1}-S_{0}$ spectrum at longer wavelengths was more open, and therefore is more amenable to analysis. This system consists of two clusters of bands that are separated by a quantum of the $\mathrm{C}=\mathrm{S}$ stretching mode, $Q_{6}$. The dense and congested band cluster that is located in the region of the $0_{0}^{0}$ transition was attributed to the activity of methyl torsion, mode $Q_{15}$. The appearance of this mode in the spectrum is somewhat unusual. On Franck-Condon grounds the long progressions in $Q_{15}$ would be the result of a twisting of the methyl group about the $\mathrm{C}-\mathrm{Me}$ axis. That is, the methyl group in the excited $T_{1}$ state must assume a molecular conformation that is different from its $S_{0}$ ground electronic state.

The assignment of the quantum numbers to the torsional fine structure was not straightforward. The initial approach to the problem was to synthesize the torsional spectrum from the ground state potential of Kroto et al..$^{9}$ (which was derived from the rotational line splittings in the microwave spectrum) and from estimates of the torsional potential for the $T_{1}$ state. In this way it was possible to identify several of the key hot bands in the spectrum and through ground state combination differences, the origin of the system as a weak band at $16292.8 \mathrm{~cm}^{-1}$. The analyses of the hot band structure led to a set of torsional levels for the lower $S_{0}$ state that were in agreement with those derived from the microwave data. The potential barrier in $V(\Theta)$ of $549.8 \mathrm{~cm}^{-1}$ derived from the ground state rotational line splittings compares favorably with the 534.3 
$\mathrm{cm}^{-1}$ value obtained from the hot band intervals observed in the visible spectrum. The torsional levels attached to the $T_{1}$ triplet state were found to be highly irregular and could not be fitted to the levels of a simple one-dimensional oscillator. The interval spacings observed in the $\mathrm{CH}_{3} \mathrm{CDS}$ spectra pointed to the source of the difficulty. The effect of deuterium substitution CDS, on the CHS frame of the molecule should have only a small effect on the $\mathrm{CH}_{3}$ torsional dynamics. The differences in the band structure which are observed in the $\mathrm{CH}_{3} \mathrm{CHS}$ and $\mathrm{CH}_{3} \mathrm{CDS}$ spectra can only be brought about by perturbations that result from interactions between methyl torsion, mode $Q_{15}^{\prime}$, and the aldehyde wagging mode, $Q_{14}^{\prime}$. In the $T_{1}$ electronic state, thioacetaldehyde bears the electron configuration $(n)^{1}\left(\pi^{*}\right)^{1}$. One of the consequences of populating the antibonding $\pi^{*}$ orbital is that molecule distorts into a pyramidal structure with the aldehyde hydrogen adopting a nonplanar equilibrium configuration. As a result, the potential function governing the out-of-plane displacement of the $\mathrm{CH}$ aldehyde bond contains a central barrier and $Q_{14}^{\prime}$ becomes a large amplitude low frequency motion. It is the coupling between the low frequency large amplitude torsional and wagging modes, $Q_{15}^{\prime}$ and $Q_{14}^{\prime}$, which is believed to be responsible for the erratic behavior of the torsional levels of the $T_{1}$ state.

For these reasons, we decided to reopen the question of the dynamics of the inversion and wagging modes in the two states. Potential surfaces $V(\alpha, \Theta)$ were generated by fitting a double Fourier expansion in $\alpha$ (the out-of-plane wagging coordinate) and $\Theta$ (methyl internal rotation) to the energy points obtained from $a b$ initio SCF calculations. Torsional-wagging manifolds for the $S_{0}$ and $T_{1}$ states were then extracted from the two-dimensional Hamiltonian $H(\alpha, \Theta)$ by a variational procedure. ${ }^{10}$ For both the wagging and the torsional modes an extended set of free rotor wave functions was used as basis functions. A torsionalwagging spectrum was derived from the calculated energy levels and the Franck-Condon factors and was compared to the observed absorption spectrum.

\section{THEORY}

In the present paper, the torsional motion arising from the methyl group and the out of plane wagging of the aldehyde hydrogen atom will be considered in the same way, i.e., the torsion as a threefold rotation around the $\mathrm{C}-\mathrm{CH}_{3}$ bond, and the wagging as a rotation of the hydrogen around an imaginary axis perpendicular to the $\mathrm{C}-\mathrm{H}$ bond lying in the CCS molecular plane and passing through the aldehyde carbon atom.

In the following discussion we shall use the formalism of Altmann ${ }^{11,12}$ to describe the nonrigid rotational operations rather than the theory of Longuet-Higgins. ${ }^{13}$

The Hamiltonian operator for the double hindered rotation in the rigid and symmetric rotor approximation can be written as

$\mathrm{H}(\alpha, \Theta)=-B_{\alpha} \frac{\partial^{2}}{\partial \alpha^{2}}-2 B_{\alpha, \Theta} \frac{\partial^{2}}{\partial \alpha \partial \theta}-B_{\Theta} \frac{\partial^{2}}{\partial \theta^{2}}+V(\alpha, \Theta)$,

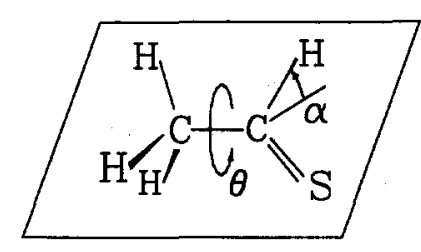

THE $\alpha$ AND $\theta$ ANGLES
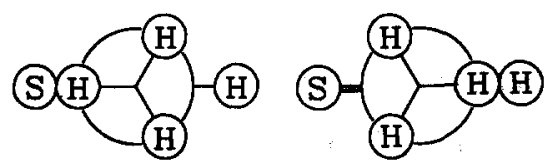

$S_{0}$ (ECLIPSED) $\quad T_{1}$ (ANTIECLIPSED)

FIG. 1. The internal rotation angle $\Theta$ and the hydrogen wagging angle $\alpha$ in thioacetaldehyde. The molecular conformations for the $S_{0}$ and $T_{1}$ electronic states: $S_{0}$, eclipsed $(\alpha=0, \Theta=0), T_{1}$ antieclipsed, $(\alpha=0, \Theta=60)$.

where $B_{\Theta}, B_{\alpha}$, and $B_{\alpha \Theta}$ are the internal rotation constants and the kinetic interaction term, respectively, and $V(\alpha, \Theta)$ is the potential energy function. The angle $\Theta$ that is defined in Fig. 1 measures the rotation of the methyl group ( $Q_{15}$ mode). The wagging motion at the aldehyde end of the molecule $\left(Q_{14}\right)$ was approximated as a rotation of the $\mathrm{CH}$ bond about an axis that lay in the CCS plane at right angles to the $\mathrm{CH}$ bond direction. The internal coordinate $\alpha$ is the angle between the $\mathrm{CH}$ bond and the CCS plane. This model is somewhat unusual in that it assumes that the aldehyde hydrogen undergoes a $C_{1}$ rotation around the $\mathrm{CH}_{3} \mathrm{CS}$ fragment.

The existence of symmetry planes in both rotors and in the frame of thioacetaldehyde allows the nonrigid group for these molecules to be expressed as ${ }^{14-18}$

$$
S=\left(C_{3}^{I} \times C_{1}^{I}\right) \wedge V^{I} \sim C_{3 v} .
$$

The subgroups $C_{3}^{I}$ and $C_{1}^{I}$ are defined as follows:

$$
C_{3}^{I}=\left[\hat{E}+\hat{C}_{3}+\widehat{C}_{3}^{2}\right] \text { or } C_{1}^{I}=[\hat{E}],
$$

while the subgroup $V^{l}$ has the form

$$
V^{I}=[\hat{E}+\hat{V}],
$$

where $\widehat{C}_{3}$ is the threefold rotational operator and $\widehat{V}$ is the double switch operator that rotates the methyl group from $\Theta$ to $-\Theta$ and simultaneously displaces the aldehyde hydrogen atom from $\alpha$ to $-\alpha$ such that

$$
\widehat{V} f(\alpha, \Theta) \equiv f(-\alpha,-\Theta) .
$$

$S$ is a nonrigid group that is isomorphic to the symmetry point group $C_{3 v}$. From the character tables, the symmetry eigenvectors corresponding to the irreducible representations of the nonrigid point group can be easily deduced on the free double rotor function basis, 
$\chi a_{1}=\cos 3 K \Theta \cos L \alpha$ and $\sin 3 K \Theta \sin L \alpha$, $\chi a_{2}=\cos 3 K \Theta \sin L \alpha$ and $\sin 3 K \Theta \cos L \alpha$, $\chi e=\cos (3 K \pm 1) \Theta \cos L \alpha$ and $\sin (3 K \pm 1) \Theta \sin L \alpha$, $\chi e=\cos (3 K \pm 1) \Theta \sin L \alpha$ and $\sin (3 K \pm 1) \Theta \cos L \alpha$.

The potential energy can be developed in terms of the $a_{1}$ symmetry eigenvectors,

$$
\begin{aligned}
V(\alpha, \Theta)= & \sum_{K, L}\left[A_{K L}^{c c} \cos K \Theta \cos L \alpha\right. \\
& \left.+A_{K L}^{s s} \sin 3 K \Theta \sin L \alpha\right] .
\end{aligned}
$$

Because of the conditions on the limits of the sine function, one term more in $K$ and $L$ in the cosine expansion has to be returned and a minimal Fourier expansion of 10 terms for the potential energy can be put forward. ${ }^{18-20}$

$$
\begin{aligned}
V(\alpha, \Theta)= & \sum_{K=0}^{2} \sum_{L=0}^{2}\left[A_{K L}^{c c} \cos 3 K \Theta \cos L \alpha\right] \\
& +A_{11}^{s s} \sin 3 \Theta \sin \alpha .
\end{aligned}
$$

As is well known, the rotational constants in the double rotor problem are related to the reduced moments of inertia of the rotors by the expressions ${ }^{21}$

$$
B_{\Theta}=\frac{\hbar^{2}}{2} \frac{I_{\alpha}}{I_{\Theta} I_{\alpha}-\Lambda_{\alpha \Theta}^{2}} \quad \text { and } \quad B_{\alpha \Theta}=\frac{\hbar^{2}}{2} \frac{\Lambda_{\alpha \Theta}}{I_{\alpha} I_{\Theta}-\Lambda_{\alpha \Theta}^{2}} ;
$$

a similar expression can be written for $B_{\alpha} . I_{\Theta}$ is the reduced moment of inertia of the rotor defined as

$$
I_{\Theta}=A_{\Theta}\left(1-\sum_{g} \lambda_{g}^{2} A_{\Theta} / M_{g}\right), \quad g=a, b, c .
$$

$A_{\Theta}$ is the moment of inertia of the internal top and $\lambda_{g}$ is the cosine of the angle between the axis of the internal top and the $g^{\text {th }}$ principal axis of the molecule. $M_{g}$ are the principal moments of inertia of the whole molecule.

$$
\Lambda_{\alpha \Theta}=\sum_{g} \frac{A_{\alpha} A_{\Theta} \lambda_{g \alpha} \lambda_{g \Theta}}{M_{g}}, \quad g=a, b, c .
$$

The solutions of the Hamiltonian operator (1) are developed, as in the one-dimensional case, onto the basis of the symmetry eigenvectors (6), which factorize the Hamiltonian matrix into boxes.

Transition intensities, $I_{n m}$, between the $T_{1}$ upper and $S_{0}$ lower torsional states $n$ and $m$, were evaluated by the relationship

$$
I_{n m} \propto g \widetilde{v}\left\langle\psi_{n} \mid \psi_{m}\right\rangle^{2} \exp \left(-E_{m} / K T\right),
$$

where $g$ is the statistical weight, $\tilde{v}=E_{n}-E_{m}$ the length of the transition (in $\mathrm{cm}^{-1}$ ), $\left\langle\psi_{n} \mid \psi_{m}\right\rangle$ the Franck-Condon factor that was calculated from the eigenvectors for the two torsional states, and $E_{m}$ the torsional energy, relative to the $S_{0}$ zero point level. For $\mathrm{CH}_{3} \mathrm{CHS}$ and $\mathrm{CH}_{3} \mathrm{CDS}$ the $a_{1}$ and $a_{2}$ levels were given nuclear statistical weights ${ }^{22}$ of 8 , while the $e$ levels were weighted 4 . The corresponding weights for $\mathrm{CD}_{3} \mathrm{CHS}$ and $\mathrm{CD}_{3} \mathrm{CDS}$ were 11 and 8 .
TABLE I. Structural parameters for the $S_{0}$ and $T_{1}$ electronic states of thioacetaldehyde.

\begin{tabular}{ccc}
\hline \hline Parameter $^{\mathrm{a}}$ & $\widetilde{\boldsymbol{X}}^{1} A^{\prime}\left(S_{0}\right)^{\mathrm{b}}$ & $\tilde{a}^{3} A^{\prime \prime}\left(T_{1}\right)^{\mathrm{c}}$ \\
\hline$r\left(\mathrm{C}-\mathrm{H}_{m}\right)$ & 1.098 & 1.098 \\
$r\left(\mathrm{C}-\mathrm{H}_{a}\right)$ & 1.089 & 1.089 \\
$r(\mathrm{C}-\mathrm{C})$ & 1.506 & 1.506 \\
$r(\mathrm{C}=\mathrm{S})$ & 1.610 & 1.799 \\
$\beta\left(\mathrm{CCH}_{m}\right)$ & 110.12 & 110.12 \\
$\beta\left(\mathrm{CCH}_{a}\right)$ & 119.40 & 119.40 \\
$\beta(\mathrm{CCS})$ & 125.28 & 121.51 \\
$\alpha($ wag $)$ & 0.00 & 20.00 \\
\hline \hline
\end{tabular}

${ }^{a}$ In $\AA$ and degrees.

${ }^{\text {bKroto }}$ et al., Ref. 9.

'Smeyers et al., Ref. 20.

The potential energy terms for methyl torsion $V(\Theta)$ were evaluated by the standard Hartree-Fock method, with the Monstergauss program. ${ }^{23}$ A 4-31G basis was employed in which six $d$-type Gaussian orbitals with exponent $a=0.39$ were centered on the sulphur atom. The restricted Hartree-Fock (RHF) and unrestricted HartreeFock (UHF) schemes were used for the singlet ground state and triplet excited states, respectively. The geometrical parameters of Table I derived from the microwave spectrum, ${ }^{9}$ were used for the lower state. The geometry of the triplet state was optimized in three directions: along the $\mathrm{C}=\mathrm{S}$ bond, across the $C C=S$ angle, and with respect to $\alpha$, the $\mathrm{CH}_{\text {ald }}$ out of plane angle. The refined values for these coordinates ${ }^{20}$ were, respectively, $1.799 \AA .121 .51^{\circ}$, and $20^{\circ}$. The total (electronic + nuclear) energies were determined for various conformations for both the singlet and triplet states, and were fitted by means of a least squares procedure to the symmetry adapted potential energy functions (8).

The internal rotation constants $B_{\alpha}, \quad B_{\Theta}$, and $B_{\alpha, \Theta}$ for methyl rotation and aldehyde wagging in the $S_{0}$ and $T_{1}$ states were calculated from Eqs. (10) and (11) and are given in Table II. The reduced moment of inertia of $B_{\Theta}$ $=7.137 \mathrm{~cm}^{-1}$, derived from the structural parameters of Table I was found to compare favorably with the 6.9950 $\mathrm{cm}^{-1}$ experimental value. ${ }^{9}$

A cross section cut out of the two-dimensional potential surface for the case of the planar frame $\left(\alpha=0.0^{\circ}\right)$ for the $S_{0}$ and $T_{1}$ states is illustrated in Fig. 2.

For the $S_{0}$ state this potential took the form

TABLE II. The internal rotation constants of thioacetaldehyde in the $S_{0}$ and $T_{1}$ states (in $\mathrm{cm}^{-1}$ ).

\begin{tabular}{lcrcc}
\hline \hline & & \multicolumn{1}{c}{$B_{\alpha}$} & $B_{\Theta}$ & $B_{\alpha \Theta}$ \\
\hline $\mathrm{CH}_{3} \mathrm{CHS}$ & $S_{0}$ & 16.615 & 7.137 & -2.079 \\
& $T_{1}$ & 16.188 & 6.695 & -1.639 \\
$\mathrm{CH}_{3} \mathrm{CDS}$ & $S_{0}$ & 9.102 & 6.819 & -1.719 \\
& $T_{1}$ & 8.773 & 6.470 & -1.376 \\
$\mathrm{CD}_{3} \mathrm{CHS}$ & $S_{0}$ & 16.401 & 4.320 & -1.927 \\
& $T_{1}$ & 15.997 & 3.897 & -1.510 \\
$\mathrm{CD}_{3} \mathrm{CDS}$ & $S_{0}$ & 8.923 & 4.031 & -1.603 \\
& $T_{1}$ & 8.610 & 3.696 & -1.276 \\
\hline \hline
\end{tabular}




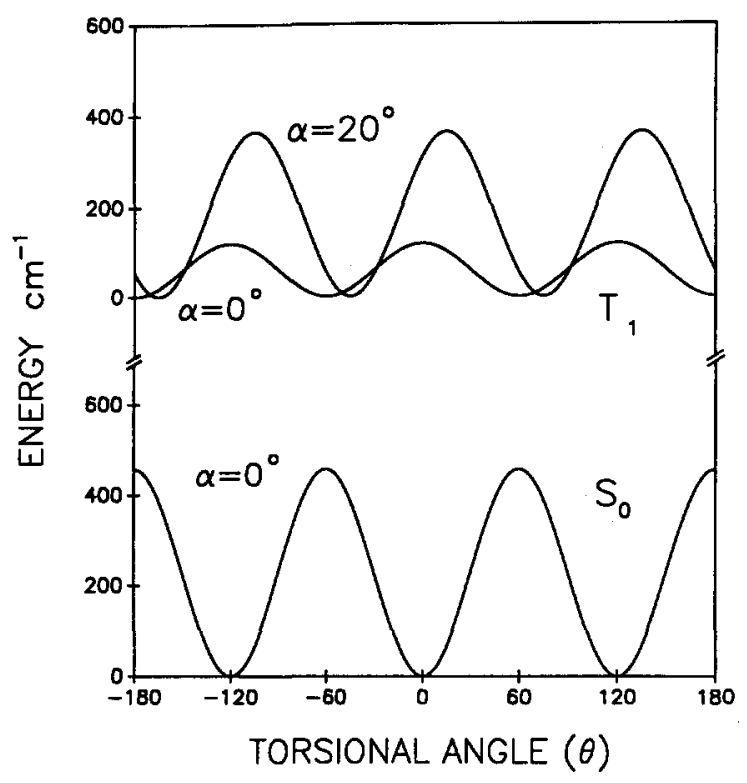

FIG. 2. Torsional potential functions for the $S_{0}$ and $T_{1}$ states of thioacetaldehyde. $\widetilde{X}^{1} A^{\prime} ; V(\alpha=0, \Theta)=229.0173-229.0173 \cos 3 \Theta, \widetilde{a}^{3} A^{\prime \prime}$; $V(\alpha=0, \Theta)=59.3480+59.3480 \cos 3 \Theta ; \quad V(\alpha=20, \Theta)$ $=182.3609+128.0594 \cos 3 \Theta+129.8378 \sin 3 \Theta$.

$V(\alpha=0, \Theta)=229.0173-229.0173 \cos 3 \Theta \mathrm{cm}^{-1}$.

The calculated barrier height of $458.0 \mathrm{~cm}^{-1}$ is in good agreement with the experimental values obtained from the microwave $^{9}\left(549.8 \mathrm{~cm}^{-1}\right)$ and visible ${ }^{7}\left(534.3 \mathrm{~cm}^{-1}\right)$ spectra. The corresponding function for the excited state,

$$
V(\alpha=0, \Theta)=59.3480+59.3480 \cos 3 \Theta \mathrm{cm}^{-1}
$$

(barrier height of $118.8 \mathrm{~cm}^{-1}$ ), may be compared to the function derived from the visible spectrum, ${ }^{5}$ where the barrier was estimated to be $94.18 \mathrm{~cm}^{-1}$. The interesting aspect of Fig. 2 is the phase relationship between the two states. It is apparent from this figure, that while a minimum in $V(\Theta)$ occurs at $\Theta=0^{\circ}$ in the lower state, the minimum in the upper state lies $60^{\circ}$. That is, thioacetaldehyde in the $S_{0}$ state adopts a conformation in which the methyl hydrogens eclipse the sulphur atom, whereas in the $T_{1}$ state the conformation is antieclipsed with the methyl group rotated by $60^{\circ}$ with respect to the lower state. Figure 1 shows the Newman projections.

The Schrödinger equations corresponding to the onedimensional Hamiltonian operator were solved variationally, for both states by expanding the solutions in terms of the free rotor solutions according to Ref. 24 . The potential functions for the two states came from Eqs. (13) and (14). The internal rotation constants came from $\mathrm{Eq}$. (9) with $\Lambda_{\alpha \Theta}=0.0$. As the matrices that require diagonalization are large (in the two-dimensional case exceeding 400 by 400 ) it was necessary to establish the minimum basis length. This is described in Table III where it is seen that while the free rotor functions are a good basis for describing methyl torsion (particularly in the $T_{1}$ state), many torsional levels are thermally populated in the lower electronic state. For this reason, it was necessary to extend the basis set to at least 37 terms for the torsional coordinate.

The torsional levels for the $S_{0}$ and $T_{1}$ states are given in Table IV. When the barrier is high, the levels form a stack with uniform spacing and may be classified by a set of torsional vibrational quantum numbers $v=0,1,2, \ldots$. The triple minimum nature of the potential leads to a tripling of the states. In order of increasing energy the $0,1,2, \ldots$ levels

TABLE III. The effect of the free rotor basis length on the levels of aldehyde wagging and methyl torsion of thioacetaldehyde in the $\bar{X} A^{\prime}\left(S_{0}\right)$ and $\widetilde{a}^{3} A^{\prime \prime}\left(T_{1}\right)$ states.

\begin{tabular}{|c|c|c|c|c|c|c|c|c|}
\hline \multicolumn{9}{|c|}{ Aldehyde wagging mode, $Q_{14}^{b}$} \\
\hline \multicolumn{4}{|c|}{$\tilde{X}^{1} A^{\prime}\left(S_{0}\right)$} & & \multicolumn{4}{|c|}{$\tilde{a}^{3} A^{\prime \prime}\left(T_{1}\right)$} \\
\hline$n^{\mathbf{c}}$ & 23 & 27 & 35 & 39 & 19 & 23 & 27 & 31 \\
\hline$a^{\prime}$ & 0.00 & 0.00 & 0.00 & 0.00 & 0.00 & 0.00 & 0.00 & 0.00 \\
\hline$a^{\prime \prime}$ & 890.50 & 842.30 & 828.02 & 827.77 & 170.07 & 168.42 & 168.22 & 168.22 \\
\hline$a^{\prime}$ & 1930.27 & 1734.48 & 1653.46 & 1651.00 & 464.64 & 463.94 & 462.37 & 462.31 \\
\hline$a^{\prime \prime}$ & 3214.20 & 2745.49 & 2484.69 & 2470.99 & 833.92 & 799.20 & 797.90 & 797.03 \\
\hline \multicolumn{9}{|c|}{ Methyl torsion mode, $Q_{15}^{\mathrm{d}}$} \\
\hline$n^{c}$ & 13 & 19 & 25 & 31 & 13 & 19 & 25 & 31 \\
\hline$a_{1}$ & 0.00 & 0.00 & 0.00 & 0.00 & 0.00 & 0.00 & 0.00 & 0.00 \\
\hline$e$ & 3.43 & 0.14 & 0.02 & 0.02 & 1.40 & 1.30 & 1.30 & 1.30 \\
\hline$e$ & 155.45 & 151.48 & 150.65 & 150.62 & 61.06 & 60.80 & 60.80 & 60.80 \\
\hline$a_{2}$ & 169.45 & 151.63 & 151.37 & 151.37 & 76.43 & 76.38 & 76.38 & 76.38 \\
\hline$a_{1}$ & 284.48 & 274.87 & 274.48 & 274.47 & 98.84 & 98.79 & 98.79 & 98.79 \\
\hline$e$ & 329.23 & 286.41 & 282.74 & 282.65 & 135.89 & 132.09 & 132.06 & 132.06 \\
\hline$e$ & 406.84 & 373.37 & 369.89 & 369.80 & 192.02 & 188.69 & 188.67 & 188.67 \\
\hline$a_{1}$ & 450.16 & 415.22 & 413.22 & 413.19 & 262.20 & 259.27 & 259.25 & 259.25 \\
\hline$a_{1}$ & 469.37 & 440.54 & 438.77 & 438.74 & 262.40 & 259.47 & 259.46 & 259.46 \\
\hline
\end{tabular}

In $\mathrm{cm}^{-1}$.

${ }^{\mathrm{b}}$ From Eqs. (15) and (16); $B_{\alpha}\left(S_{0}\right)=16.010, B_{\alpha}\left(T_{1}\right)=15.787$.

'Total number of basis functions.

${ }^{d}$ From Eqs. (13) and (14); $B_{\Theta}\left(S_{0}\right)=6.877, B_{\Theta}\left(T_{1}\right)=6.529$. 
TABLE IV. A comparison of the torsional levels for the $S_{0}$ and $T_{1}$ states of thioacetaldehyde in one and two dimensions. ${ }^{\mathrm{a}}$

\begin{tabular}{|c|c|c|c|c|c|c|}
\hline \multirow[b]{2}{*}{$v$} & \multirow[b]{2}{*}{ sym. } & \multicolumn{2}{|c|}{$S_{0}$} & \multicolumn{3}{|c|}{$T_{1}$} \\
\hline & & $V\left(\alpha=0^{\circ}, \Theta\right)$ & $V(\alpha, \Theta)$ & $V\left(\alpha=0^{\circ}, \Theta\right)$ & $V\left(\alpha=20^{\circ}, \Theta\right)$ & $V(\alpha, \Theta)$ \\
\hline 0 & $a_{1}$ & 0.00 & 0.00 & 0.00 & 0.00 & 0.00 \\
\hline 0 & $e$ & 0.02 & 0.03 & 1.30 & 0.18 & 0.57 \\
\hline 1 & $e$ & 150.65 & 141.78 & 60.80 & 103.90 & 63.73 \\
\hline 1 & $a_{2}$ & 151.39 & 142.67 & 76.37 & 107.85 & 68.38 \\
\hline 2 & $a_{1}$ & 274.49 & 259.93 & 98.79 & 174.63 & 132.50 \\
\hline 2 & $e$ & 282.68 & 268.89 & 132.05 & 194.04 & 151.21 \\
\hline 3 & $e$ & 369.83 & 353.59 & 188.65 & 249.68 & 192.66 \\
\hline 3 & $a_{2}$ & 413.25 & 398.43 & 259.22 & 313.18 & 280.82 \\
\hline \multicolumn{2}{|c|}{ Barrier ht. } & 458.0 & 458.0 & 118.9 & 364.7 & $239.2^{\mathrm{b}}$ \\
\hline
\end{tabular}

${ }^{\mathrm{a}}$ In $\mathrm{cm}^{-1}$

'Saddle point.

divide into pairs of microstates $a_{1} e, e a_{2}, a_{1} e, \ldots$. Selection rules allow the torsional states to combine as $a_{1} \leftrightarrow a_{1}, a_{2} \leftrightarrow a_{2}, e \leftrightarrow e$. The notation for designating the transitions follows the usual convention in which the active mode is subscripted and superscripted with the appropriate quantum numbers. As a consequence the origin band, which is commonly written $0_{0}^{0}$, consists of two bands: $0_{0}^{0} a_{1}$ and $0_{0}^{0} e$. For example, $14_{0}^{1} 15_{0}^{2} e$ would be the designation for an $e \leftrightarrow e$ transition between the zero point levels of the two states where one quantum of aldehyde hydrogen wagging $Q_{14}$ and two quanta of methyl torsion $Q_{15}$ are excited.

Calculated spectra for $\mathrm{CH}_{3} \mathrm{CHS}$, with $T=300 \mathrm{~K}$, are shown in Fig. 3 where the position of the $0_{0}^{0} a_{1}$ transition is scaled to zero $\mathrm{cm}^{-1}$. The torsional spectrum which was

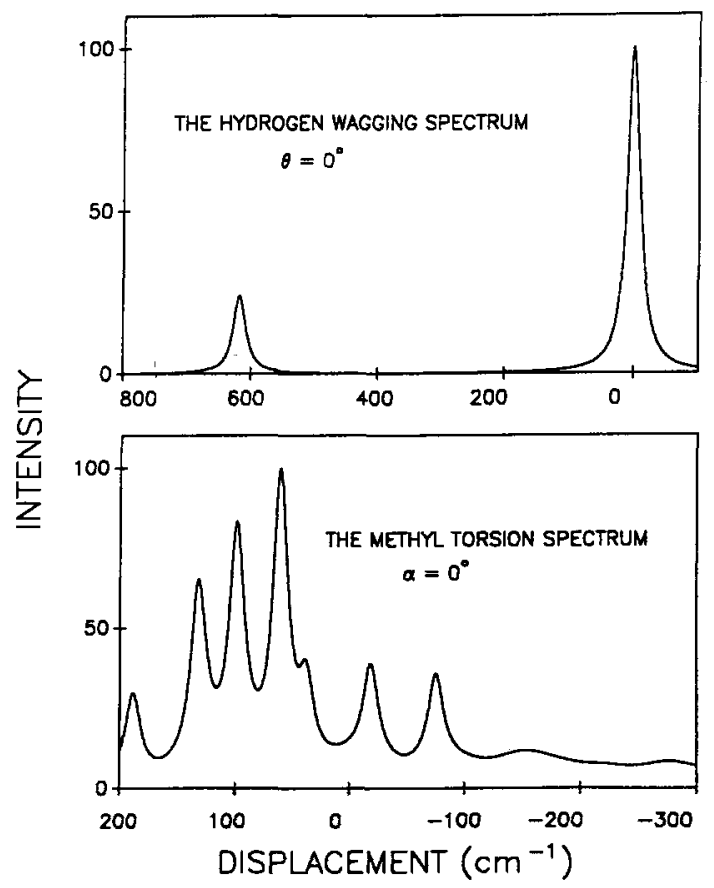

FIG. 3. Calculated hydrogen wagging and methyl torsion spectra of thioacetaldehyde with the approximation that the modes are uncoupled. derived from the $a b$ initio potential functions of Eqs. (13) and (14) gives an overall representation of the torsional structure that is similar to the previous study, ${ }^{7}$ which was obtained from a direct fit to the observed band positions. The hot band structure, in the region 0 to $-400 \mathrm{~cm}^{-1}$, which emanates from the higher torsional levels of the $S_{0}$ state is well accounted for. On the other hand, the bands that come from transitions that terminate on the higher levels of the $T_{1}$ state, 0 to $+400 \mathrm{~cm}^{-1}$, are poorly described by this one-dimensional approach based on $a b$ initio calculations.

The energy terms for the hydrogen wagging $V(\alpha)$ potential were evaluated in the same way as those of $V(\Theta)$. Higher terms $A_{2}^{c} \cos 2 \alpha$ and $A_{3}^{c} \cos 3 \alpha$ were added to the potential to give it the quadratic form expected for the lower electronic state, or to shape it into the broad double minimum function of the upper state. The least squares fit of the potential energy to the data points derived from $a b$ initio calculations gave for the $S_{0}$ state,

$$
\begin{aligned}
V(\alpha, \Theta=0)= & 20663.6331-20327.4647 \cos \alpha \\
& -336.1684 \cos 2 \alpha \mathrm{cm}^{-1}
\end{aligned}
$$

and for the $T_{1}$ state with eclipsed conformation, $\Theta=0^{\circ}$,

$$
\begin{aligned}
V(\alpha, \Theta=0)= & 9429.7243-10262.3033 \cos \alpha \\
& -493.8151 \cos 2 \alpha \\
& +1326.3940 \cos 3 \alpha \mathrm{cm}^{-1}
\end{aligned}
$$

The potential functions for hydrogen wagging in the $S_{0}$ and $T_{1}$ states are illustrated in Fig. 4. It is clear that the dynamics in the two states are very different. As would be expected, the lower function has the quadratic form of a harmonic oscillator. The upper function, on the other hand, is broad and flat over $\pm 30^{\circ}$ and does not possess a central barrier. When the methyl $\mathbf{C}-\mathrm{H}$ bond is allowed to antieclipse the thiocarbonyl group, $\Theta=60^{\circ}$, the potential function becomes 


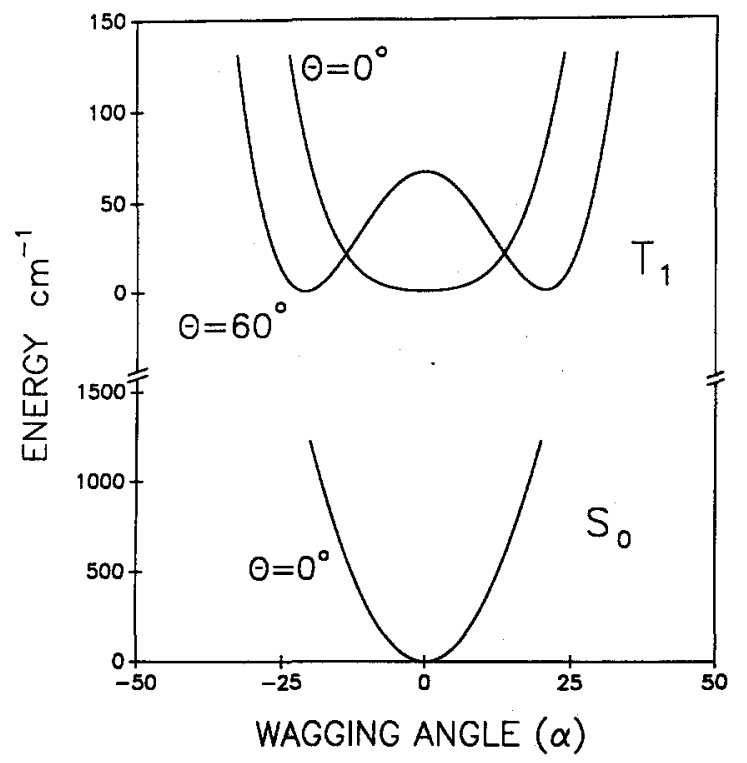

FIG. 4. Hydrogen wagging potential functions for the $S_{0}$ and $T_{1}$ states of thioacetaldehyde. $\widetilde{X}^{1} A^{\prime} ; V(\alpha, \Theta=0)=20663.6331$ $-20327.4647 \cos \alpha-336.1684 \cos 2 \alpha, \quad \tilde{a}^{3} A^{\prime \prime} ; V(\Theta=0, \alpha)$ $=9429.7243-10262.3033 \cos \alpha-493.8151 \cos 2 \alpha+1326.3940$ $X \cos 3 \alpha ; V(\alpha, \Theta=60)=9548.4203-11307.4070 \cos \alpha+493.8151$ $\times \cos 2 \alpha+1265.1716 \cos 3 \alpha$.

$$
\begin{aligned}
V(\alpha, \Theta=60)= & 9548.4203-11307.4070 \cos \alpha \\
& +493.8151 \cos 2 \alpha \\
& +1265.1716 \cos 3 \alpha \mathrm{cm}^{-1}
\end{aligned}
$$

This function, which is shown in Fig. 4, is broad and flat, but in this case has a barrier at $\alpha=0^{\circ}$ of $76.7 \mathrm{~cm}^{-1}$ with minima at $\pm 20^{\circ}$.

With this value of the aldehyde hydrogen equilibrium angle, it is possible to return to the problem of the methyl torsion. A better approximation to the dynamics of the torsional motion would be to calculate the torsional potential function with the wagging coordinate fixed at its equilibrium value, $\alpha=20.0^{\circ}$. When this is done the potential function becomes

$$
\begin{aligned}
V(\alpha=20, \Theta)= & 182.3609+128.0594 \cos 3 \Theta \\
& +129.8378 \sin 3 \Theta \mathrm{cm}^{-1} .
\end{aligned}
$$

This function, which is shown in Fig. 2, has a barrier height of $365.0 \mathrm{~cm}^{-1}$ and minima at $75^{\circ}, 75-120^{\circ}$, and $75-240^{\circ}$. The height of the barrier to methyl torsion is sensitive to the position of the aldehyde hydrogen. It undergoes a threefold increase from 118.8 to $365.0 \mathrm{~cm}^{-1}$ as $\alpha$ goes from $0.0^{\circ}$ to $20^{\circ}$.

The hydrogen wagging spectrum derived from the potential functions (15) and (16) is illustrated in Fig. 3. It shows the effect of the selection rules for the $C_{s}$ nonrigid group (clamped methyl torsion) in that the transition $14_{0}^{2}$ is allowed, $a^{\prime} \leftrightarrow a^{\prime}$, and appears as a weak band in the spectrum whereas the single quantum addition $14_{0}^{1} a^{\prime} \leftrightarrow a^{\prime \prime}$, is forbidden and is absent. The calculated levels are shown in Table V. The level structure for the $S_{0}$ state is
TABLE V. Calculated energy levels ${ }^{\mathrm{a}}$ for the aldehyde wagging motion in the $S_{0}$ and $T_{1}$ states of thioacetaldehyde (one-dimensional case only). ${ }^{b}$

\begin{tabular}{ccccc}
\hline \hline$v$ & sym. & $S_{0}: V\left(\alpha, \Theta=0^{\circ}\right)^{\mathrm{c}}$ & $T_{1}: V\left(\alpha, \Theta=0^{\circ}\right)^{\mathrm{d}}$ & $T_{1}: V\left(\alpha, \Theta=60^{\circ}\right)^{\mathrm{c}}$ \\
\hline 0 & $a^{\prime}$ & 0.00 & 0.00 & 0.00 \\
1 & $a^{\prime \prime}$ & 828.38 & 270.44 & 168.30 \\
2 & $a^{\prime}$ & 1654.17 & 616.28 & 462.52 \\
3 & $a^{\prime \prime}$ & 2485.71 & 1003.04 & 797.38 \\
4 & $a^{\prime}$ & 3344.63 & 1420.44 & 1170.57 \\
5 & $a^{\prime \prime}$ & 4264.96 & 1861.96 & 1572.53 \\
\multicolumn{7}{c}{ Barrier ht. } & 0.0 & 0.0 & 76.7 \\
\hline \hline
\end{tabular}

In $\mathrm{cm}^{-1}$.

'Total of 35 basis functions; $18 a^{\prime}+17 a^{\prime \prime}$.

${ }^{\mathrm{c}} V\left(\alpha, \Theta=0^{\circ}\right)=20663.6331-20327.4647 \cos \alpha-336.1684 \cos 2 \alpha$.

${ }^{\mathrm{d}} V\left(\alpha, \Theta=0^{\circ}\right)=9429.7243-10262.3033 \cos \alpha-493.8151 \cos 2 \alpha$ $+1326.3940 \cos 3 \alpha$.

${ }^{\mathrm{e}} V\left(\alpha, \Theta=60^{\circ}\right)=9615.0218-11307.4070 \cos \alpha-493.8151 \cos 2 \alpha$ $+1265.1716 \cos 3 \alpha$.

well behaved and it is only for the higher $v=3$ and $v$ $=4$ quanta that inadequacies in the basis length become apparent.

Very few substituted thiocarbonyl compounds are available for spectral correlation. The out-of-plane wagging vibration for trans-dithioformic acid that has been observed ${ }^{25}$ at $824.0 \mathrm{~cm}^{-1}$ can be combined with the corresponding frequencies ${ }^{26,27}$ for $\mathrm{CH}_{3} \mathrm{CHO} / \mathrm{CHOOH}$ of $1107.3 / 1033.4 \mathrm{~cm}^{-1}$ to predict an out-of-plane frequency of $882.9 \mathrm{~cm}^{-1}$ for $\mathrm{CH}_{3} \mathrm{CHS}$. The value calculated here for $Q_{14}$ of $828.4 \mathrm{~cm}^{-1}$ is in good agreement with this prediction and gives some confidence to the validity of the molecular model. The hydrogen wagging levels for the $T_{1}$ state which are given in Table IV are calculated for the eclipsed, $\Theta=0^{\circ}$, and antieclipsed, $\Theta=60^{\circ}$, configurations. Both calculations yield levels which have reversed anharmonicity (first interval smaller than the second) with about one-third of the ground state wagging energy that is characteristic of levels contained in a broad shallow potential function. The levels are very sensitive to the conformation of the methyl group: for $\Theta=0^{\circ}$; the wagging quanta are: $270.44,616.28$, and for $\Theta=60^{\circ} ; 168.30,462.52 \mathrm{~cm}^{-1}$, which perhaps is not too surprising since both the methyl torsion and hydrogen wagging modes are low frequency large amplitude motions. The pronounced variation of the torsional level structure with the hydrogen wagging angle demonstrates that the $Q_{14}$ and $Q_{15}$ modes are strongly coupled in the upper triplet state. It follows that the correct description of the $T_{1}-S_{0}$ spectrum would require an analysis of the joint motions of methyl internal rotation and aldehyde hydrogen wagging.

The potential function, $V(\alpha, \Theta)$, which describes the joint wagging-torsional motion was obtained as a fit of the two-dimensional Fourier expansion, (8) to the calculated total energy points by $a$ linear regression procedure. ${ }^{28}$ For the $S_{0}$ state the potential took the form

$$
\begin{aligned}
V(\alpha, \Theta)= & 20633.10-20435.62 \cos \alpha \\
& +107.15 \cos 3 \Theta \cos \alpha \\
& -336.17 \cos 3 \Theta \cos 2 \alpha \\
& +657.11 \sin 3 \Theta \sin \alpha \mathrm{cm}^{-1}
\end{aligned}
$$




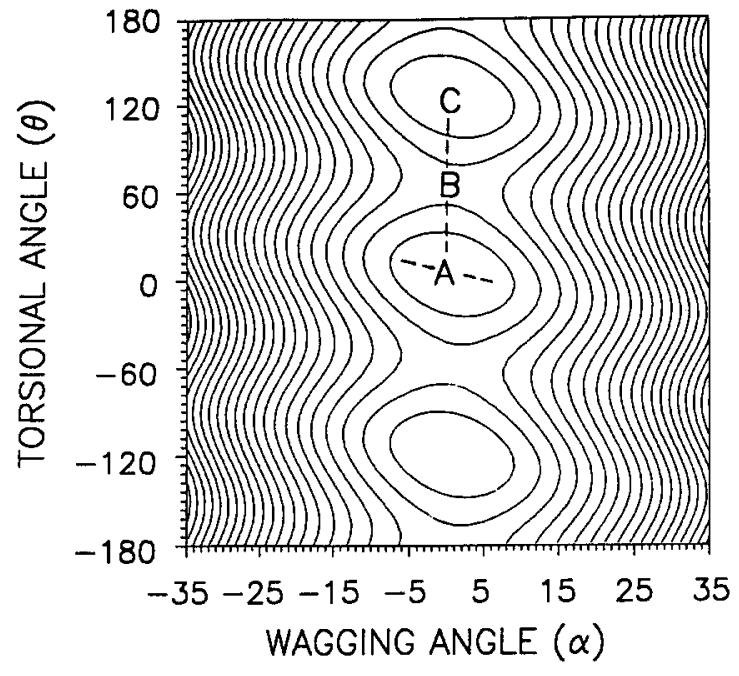

FIG. 5. The potential energy surface $V(\alpha, \Theta)$ for the $S_{0}$ ground electronic state. The interval between the isopotential lines is $200.0 \mathrm{~cm}^{-1}$.

with a correlation factor very close to one: $R=1.0000$ and a standard deviation $\sigma=10.31 \mathrm{~cm}^{-1}$.

Similarly, for the triplet state:

$V(\alpha, \Theta)=9668.92-10784.85 \cos \alpha+1295.78 \cos 3 \alpha$

$+522.55 \cos 3 \Theta \cos \alpha$

$-493.82 \cos 3 \Theta \cos 2 \alpha$

$+30.61 \cos 3 \Theta \cos 3 \alpha-92.18 \sin 3 \Theta \sin \alpha$

$+186.33 \sin 3 \Theta \sin 3 \alpha \mathrm{cm}^{-1}$,

with $R=0.9990$ and $\sigma=45.14 \mathrm{~cm}^{-1}$.

Plots of the potential surface for the $S_{0}$ and $T_{1}$ states are given in Figs. 5 and 6. The form of the motion can be understood from an examination of these surfaces. In the $S_{0}$ state, the $Q_{15}$ mode moves the methyl group from point $\mathrm{A}(\alpha=0, \Theta=0)$ to $\mathrm{B}(\alpha=0, \Theta=60)$ and then onto

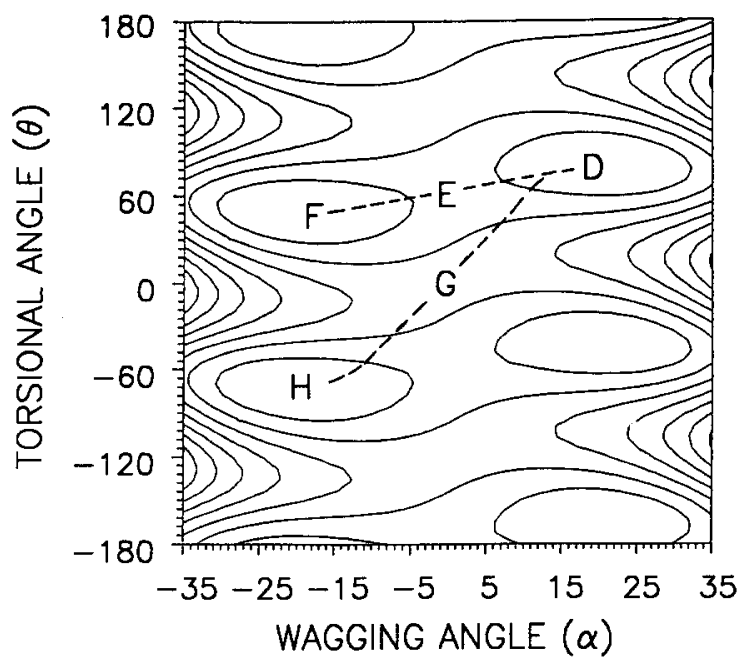

FIG. 6. The potential surface $V(\alpha, \Theta)$ for the $T_{1}$ excited electronic state. The interval between the contour lines is $100.0 \mathrm{~cm}^{-1}$.

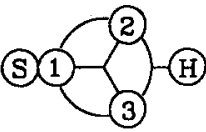

(A) $0 \mathrm{~cm}^{-1}$<smiles>CC1CCCCC12OCCCC2C</smiles>

(D) $0 \mathrm{~cm}^{-1}$

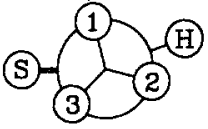

(D) $0 \mathrm{~cm}^{-1}$

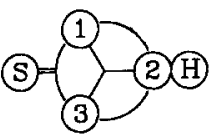

(B) $458 \mathrm{~cm}^{-1}$

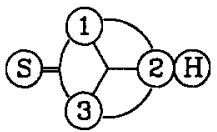

(E) $120 \mathrm{~cm}^{-1}$

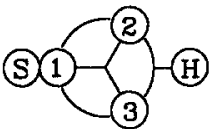

(G) $239 \mathrm{~cm}^{-1}$

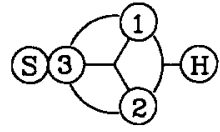

(C) $0 \mathrm{~cm}^{-1}$

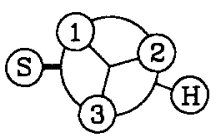

(F) $0 \mathrm{~cm}^{-1}$

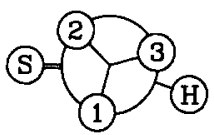

(H) $0 \mathrm{~cm}^{-1}$
FIG. 7. The molecular configurations in the $S_{0}$ and $T_{1}$ electronic states. The (A) eclipsed, (B) antieclipsed, and (C) eclipsed structures represent the conformational changes that occur in $S_{0}$ thioacetaldehyde as the methyl group rotates from point (A) to point (B) to point (C) in Fig. 5. The diagrams (D), (E), and (F) illustrate the structural changes which occur as $T_{1}$ thioacetaldehyde undergoes molecular inversion and (D), $(\mathrm{G})$, and $(\mathrm{H})$ are the corresponding changes in structure for methyl rotation.

point $\mathrm{C}(\alpha=0, \Theta=120)$, while the molecular conformation changes from an eclipsed to an antieclipsed and then back to an eclipsed conformation. The height of the barrier at point $B$ relative to equilibrium energy of point $A$ is calculated to be $458.0 \mathrm{~cm}^{-1}$, which is to be compared to the experimental value of $534.3 \mathrm{~cm}^{-1}$. Newman projection diagrams illustrating the changes in conformation are given in Fig. 7. In the lower $S_{0}$ state, the aldehyde wagging mode can be described as a high frequency low amplitude vibration which is localized in a single minimum potential. That the dotted line trajectory representing $Q_{14}^{r}$ is only slightly skewed with respect to the $\alpha$ axis indicates that the coupling between the $\Theta$ and $\alpha$ coordinates is small. The interpretation of the dynamics in the $T_{1}$ state is more complex. From the potential surface of Fig. 6 it is clear that one full cycle of the wagging-torsion coordinates generates six minima. The equilibrium configuration of point $D, \alpha=20, \Theta=75$, can be described as staggered. For wagging displacement to occur, the molecule must traverse the lowest saddle point on the surface at point $E$ where it adopts an antieclipsed conformation, $\alpha=0^{\circ}, \Theta=60^{\circ}$. This point is $120.5 \mathrm{~cm}^{-1}$ above the equilibrium position. From here the wagging mode moves onto configuration $F\left(\alpha=-20, \Theta=45^{\circ}\right)$. Note that while the wagging mode involves a $20^{\circ}$ displacement of the hydrogen from the molecular plane it has also associated with it a $15^{\circ}$ rotation of the methyl group. That is, the aldehyde wagging mode is nearly an equal admixture of the $\alpha$ and $\Theta$ internal coordinates. This motion resembles an internal rotation in the sense that the two ends of the molecule counter rotate 
TABLE VI. Calculated energy levels $\mathrm{s}^{\mathrm{a}}$ for the coupled methyl torsion mode, $Q_{15}$, and the aldehyde wagging mode, $Q_{14}$, in the $S_{0}$ and $T_{1}$ states of thioacetaldehyde: $\mathrm{CH}_{3} \mathrm{CHS}, \mathrm{CH}_{3} \mathrm{CDS}, \mathrm{CD}_{3} \mathrm{CHS}, \mathrm{CD}_{3} \mathrm{CDS}$.

\begin{tabular}{|c|c|c|c|c|c|c|c|c|c|c|}
\hline \multirow[b]{2}{*}{$v_{14}$} & \multirow[b]{2}{*}{$v_{15}$} & \multirow[b]{2}{*}{ sym. } & \multicolumn{2}{|c|}{$\mathrm{CH}_{3} \mathrm{CHS}$} & \multicolumn{2}{|c|}{$\mathrm{CH}_{3} \mathrm{CDS}$} & \multicolumn{2}{|c|}{$\mathrm{CD}_{3} \mathrm{CHS}$} & \multicolumn{2}{|c|}{$\mathrm{CD}_{3} \mathrm{CDS}$} \\
\hline & & & $S_{0}^{b}$ & $T_{1}^{\mathrm{c}}$ & $S_{0}{ }^{b}$ & $T_{1}^{\mathrm{c}}$ & $S_{0}^{\mathrm{b}}$ & $T_{1}^{\mathrm{c}}$ & $S_{0}^{b}$ & $T_{0}{ }^{\mathrm{c}}$ \\
\hline 0 & 0 & $a_{1}$ & 0.000 & 0.000 & 0.000 & 0.000 & 0.000 & 0.000 & 0.000 & 0.000 \\
\hline 0 & 0 & $e$ & 0.028 & 0.572 & 0.019 & 0.634 & 0.001 & 0.067 & 0.001 & 0.081 \\
\hline 0 & 1 & $e$ & 144.68 & 63.73 & 142.74 & 44.68 & 114.82 & 49.44 & 111.52 & 33.02 \\
\hline 0 & 1 & $a_{2}$ & 145.53 & 68.38 & 143.35 & 47.43 & 114.86 & 50.15 & 111.54 & 33.46 \\
\hline 0 & 2 & $a_{1}$ & 264.85 & 132.50 & 263.27 & 121.17 & 219.19 & 109.81 & 213.67 & 95.56 \\
\hline 0 & 2 & $e$ & 273.53 & 151.21 & 270.08 & 132.71 & 220.00 & 115.26 & 214.13 & 99.14 \\
\hline 0 & 3 & $e$ & 359.22 & 192.66 & 357.53 & 153.48 & 308.69 & 156.14 & 302.95 & 130.38 \\
\hline 0 & 3 & $a_{2}$ & 403.56 & 280.82 & 395.29 & 246.04 & 316.22 & 176.26 & 307.26 & 138.26 \\
\hline 1 & 0 & $a_{2}$ & 834.88 & 213.16 & 627.66 & 162.23 & 830.49 & 219.41 & 622.81 & 184.09 \\
\hline 1 & 0 & $e$ & 834.90 & 239.01 & 627.71 & 206.80 & 830.49 & 212.56 & 622.81 & 197.45 \\
\hline 1 & 1 & $e$ & 972.92 & 312.46 & 766.16 & 289.55 & 940.24 & 278.99 & 731.45 & 229.46 \\
\hline 1 & 1 & $a_{1}$ & 974.07 & 345.31 & 767.04 & 291.16 & 940.32 & 297.75 & 731.49 & 232.22 \\
\hline
\end{tabular}

${ }^{2}$ In $\mathrm{cm}^{-1}$. Total of 31 wagging and 37 torsional basis functions.

${ }^{\mathrm{b}} V(\alpha, \Theta)=20663.10-20435.62 \cos \alpha+107.15 \cos 3 \Theta \cos \alpha-336.17 \cos 3 \Theta \cos 2 \alpha+657.11 \sin 3 \Theta \sin \alpha$.

${ }^{\mathrm{c}} V(\alpha, \Theta)=9668.92-10784.85 \cos \alpha+1295.78 \cos 3 \alpha+522.55 \cos 3 \Theta \cos \alpha-493.82 \cos 3 \Theta \cos 2 \alpha+30.61 \cos 3 \Theta \cos 3 \alpha-92.18 \sin 3 \Theta \sin \alpha$ $+186.33 \sin 3 \Theta \sin 3 \alpha$.

against each other. The saddle point for the $Q_{15}$ methyl rotation is located at $\mathrm{G}\left(\alpha=0^{\circ}, \Theta=0^{\circ}\right)$, which is 239.2 $\mathrm{cm}^{-1}$ above D. The dotted line D-G-H represents one possible path for the rotation of the methyl group.

Following Ref. 10, the corresponding Schrödinger equations for the coupled torsion wagging Hamiltonian operator $H(\alpha, \Theta)$ were solved variationally for both electronic states. Solutions were expanded in terms of the symmetry eigenvectors (6), which factorize the Hamiltonian matrix into boxes. $B_{\alpha}, B_{\Theta}$, and $B_{\alpha, \Theta}$ were taken from Table II. It was found that 31 wagging and 37 torsional functions gave a sufficient basis length. This symmetrization reduced the $1147 \times 1147$ direct product matrix to smaller $a_{1}, a_{2}$, and $e$ blocks of dimension 202, 201, and $372+372$. Table IV gives the energy level data derived from the twodimensional Hamiltonian along with calculations in one dimension evaluated at fixed out of plane angle: $\alpha=0.0^{\circ}$ and $\alpha=20.0^{\circ}$ for the sake of comparison. Table VI gives energy levels for the four isotopomers of thioacetaldehyde.

The calculated levels for the $S_{0}$ ground electronic state are well behaved. The aldehyde wagging mode, $Q_{14}$ $=834.88 \mathrm{~cm}^{-1}$, is a small amplitude out of plane vibration of high frequency and does not couple strongly with the $Q_{15}$ methyl torsion. This can be seen from the isotope data of Table VI. In $\mathrm{CH}_{3} \mathrm{CHS}\left(S_{0}\right)$ the $Q_{15}$ mode has a value of $15_{1 e}=144.68 \mathrm{~cm}^{-1}$, which changes only slightly to 142.74 $\mathrm{cm}^{-1}$ in $\mathrm{CH}_{3} \mathrm{CDS}\left(S_{0}\right)$ when the CHS frame is deuterated. Moreover, the stack of torsional levels in $Q_{15}$ that is built on the $Q_{14}$ wagging mode has the same $a_{1}-e$ splittings as those that are attached to the zero point level $\left(14_{0} 15_{0 e}-14_{0} 15_{0 a 1}=0.028 \mathrm{~cm}^{-1} ; 14115_{0 e}-14_{1} 15_{0 a 2}\right.$ $\left.=0.021 \mathrm{~cm}^{-1}\right)$. The situation is very different in the excited triplet $T_{1}$ state since both $Q_{14}$ and $Q_{15}$ are now of large amplitude. The central two columns of Table IV give the torsional levels derived from the one-dimensional Hamiltonians $\left(\alpha=0^{\circ}\right.$, barrier height $=118.9 \mathrm{~cm}^{-1}$ and $\alpha=20^{\circ}$, barrier height $=364.7 \mathrm{~cm}^{-1}$ ). The widely differing potential functions lead to different energy levels.
Compare the $15^{1 e} / 15^{102}$ levels at $60.80 / 76.37 \mathrm{~cm}^{-1}$ for the planar case, $\alpha=0.0^{\circ}$ with the $103.90 / 107.85 \mathrm{~cm}^{-1}$ values derived for the equilibrium configuration where the out plane angle is fixed at $20^{\circ}$. These two cases represent the limiting values for the coupling and it is not too surprising that the two-dimensional calculation yields the energy levels, 63.73/68.38. The data of Table VI demonstrates just how strongly the modes are coupled in the upper electronic state. In $\mathrm{CH}_{3} \mathrm{CHS}$ the first quantum of torsion, $15^{1}$ has a value of $63.63 \mathrm{~cm}^{-1}$. On deuteration of the aldehyde group, $\mathrm{CH}_{3} \mathrm{CDS}$, this interval drops to $44.68 \mathrm{~cm}^{-1}$. Indeed the mixing is so severe that it is difficult to give a clear quantum number designation to the higher torsional levels of the triplet state. For example, while the $a_{1}-e$ splitting of the zero point level in $\mathrm{CH}_{3} \mathrm{CHS}$ is $0.572 \mathrm{~cm}^{-1}$, it increases to $25.85 \mathrm{~cm}^{-1}$ when it is attached to $v_{14}=1$. This would indicate that the methyl group undergoes nearly free rotation when quanta of torsion combine with quanta of the wagging mode.

Intensities were calculated from the Franck-Condon overlap factors and are listed along with the band positions in Table VII. Figure 8 shows the observed and calculated spectra for $\mathrm{CH}_{3} \mathrm{CHS}$ at $614 \mathrm{~nm}$ while Fig. 9 shows plots of $\mathrm{CH}_{3} \mathrm{CHS} / \mathrm{CH}_{3} \mathrm{CDS} / \mathrm{CD}_{3} \mathrm{CHS} / \mathrm{CD}_{3} \mathrm{CDS}$ where each of the calculated lines was artificially broadened. The absorption bands have a distinct contour which is the result of the overlap of many rovibronic transitions. For the sake of comparison with the experimental spectrum, it was assumed that under low resolution conditions used here the contour of each band could be simulated by a Lorentzian function. While this seems to be satisfactory for the lower bands in the spectrum, the contour of the $15_{0}^{2} e$ band appears to be quite different and to extend in a series of equally spaced subbands over a $50 \mathrm{~cm}^{-1}$ range. The agreement between the overall profiles of the observed and calculated spectra is quite good in that the major features are reproduced. Of importance is the dip in the intensity that is 
TABLE VII. Calculated transition energies, ${ }^{a}$ and intensities for the methyl torsion and aldehyde wagging modes in the $T_{1}-S_{0}$ system of thioacetaldehyde (two-dimensional case). ${ }^{\mathrm{b}}$

\begin{tabular}{|c|c|c|c|c|c|c|c|c|}
\hline \multirow[b]{2}{*}{ Assign. } & \multicolumn{2}{|c|}{$\mathrm{CH}_{3} \mathrm{CHS}$} & \multicolumn{2}{|c|}{$\mathrm{CH}_{3} \mathrm{CDS}$} & \multicolumn{2}{|c|}{$\mathrm{CD}_{3} \mathrm{CHS}$} & \multicolumn{2}{|c|}{$\mathrm{CD}_{3} \mathrm{CDS}$} \\
\hline & $\mathrm{cm}^{-1}$ & int. & $\mathrm{cm}^{-1}$ & int. & $\mathrm{cm}^{-1}$ & int. & $\mathrm{cm}^{-1}$ & int. \\
\hline $15_{0}^{2} e$ & 151.18 & 100.0 & 132.69 & 100.0 & 115.25 & 26.5 & 99.14 & 28.9 \\
\hline $15_{0}^{2} a_{1}$ & 132.50 & 93.3 & 121.17 & 74.4 & 109.81 & 37.8 & 95.56 & 43.4 \\
\hline $15_{0}^{3} e$ & 192.63 & 66.0 & 153.46 & 30.8 & 156.14 & 100.0 & 130.38 & 100.0 \\
\hline $15_{0}^{1} e$ & 63.70 & 63.8 & 44.66 & 40.8 & 49.43 & 14.7 & 33.02 & 15.7 \\
\hline $15_{0}^{4} a_{1}$ & 260.33 & 36.9 & 209.02 & 41.9 & 195.90 & 53.9 & 172.33 & 88.1 \\
\hline $15_{1}^{3} e^{0}$ & 6.52 & 33.8 & -10.03 & 12.6 & 44.47 & 34.5 & -12.37 & 39.8 \\
\hline $15 a_{2}$ & -77.15 & 31.8 & -95.91 & 21.7 & -64.71 & 14.0 & -78.08 & 16.0 \\
\hline $14{ }_{0}^{1} 15_{0}^{0} e$ & 238.98 & 31.5 & 206.79 & 71.4 & 212.56 & 22.1 & 170.47 & 81.9 \\
\hline $15_{1}^{2} e$ & 16.77 & 27.4 & 10.73 & 28.0 & 41.33 & 13.3 & 18.86 & 17.0 \\
\hline $14{ }_{0}^{1} 15_{1}^{1} e$ & 167.78 & 27.4 & 99.80 & 40.0 & 121.14 & 24.2 & 85.93 & 49.5 \\
\hline $15_{1}^{0} e$ & -144.12 & 23.5 & -142.11 & 21.5 & -114.74 & 65.9 & -111.43 & 10.3 \\
\hline $15_{2}^{i} e$ & -209.80 & 23.4 & -225.40 & 17.8 & -170.55 & 21.0 & -181.10 & 25.4 \\
\hline $15_{3}^{0} e$ & -358.64 & 23.4 & -356.90 & 20.8 & -308.61 & 17.2 & -302.86 & 23.0 \\
\hline $15_{2}^{0} a_{1}$ & -264.85 & 20.1 & -263.27 & 17.4 & -219.18 & 7.8 & -213.67 & 11.2 \\
\hline $14_{0}^{1} 15_{1}^{0} a_{2}$ & 67.6 & 15.5 & 19.01 & 20.2 & 61.40 & 26.5 & 26.72 & 28.5 \\
\hline $15_{1}^{1} e$ & -80.96 & 14.2 & -98.06 & 10.6 & -65.37 & 6.8 & -78.49 & 7.9 \\
\hline $14{ }_{0}^{1} 15_{2}^{0} e$ & -34.52 & 14.0 & -63.27 & 11.0 & -7.42 & 7.1 & -43.64 & 5.4 \\
\hline $15_{2}^{0} e$ & -272.96 & 12.6 & -269.44 & 10.6 & -219.92 & 4.0 & -214.04 & 5.7 \\
\hline $15_{1}^{3} a_{2}$ & -135.29 & 11.5 & 102.68 & 2.7 & 107.36 & 2.7 & 72.55 & 10.8 \\
\hline $0_{0}^{0} a_{1}$ & -0.00 & 10.0 & 0.00 & 10.0 & 0.00 & 1.8 & 0.00 & 3.1 \\
\hline
\end{tabular}

${ }^{a}$ Relative to $0_{0}^{0} a_{1}$.

bIn $\mathrm{cm}^{-1}$.

observed between the $15_{0}^{1} e$ and $15_{0}^{2} a_{1}$ bands in the center of the absorption spectrum. This intensity minimum is not reproduced in either of the one-dimensional calculations based on the $V(\alpha=0, \Theta)$ or $V(\alpha=20, \Theta)$ potentials.

The strongest band in the absorption spectrum, which is observed at $16402.6 \mathrm{~cm}^{-1}$, appears very clearly as a linelike feature. It is assigned to the transition $15_{0}^{2} a_{1}$ that is

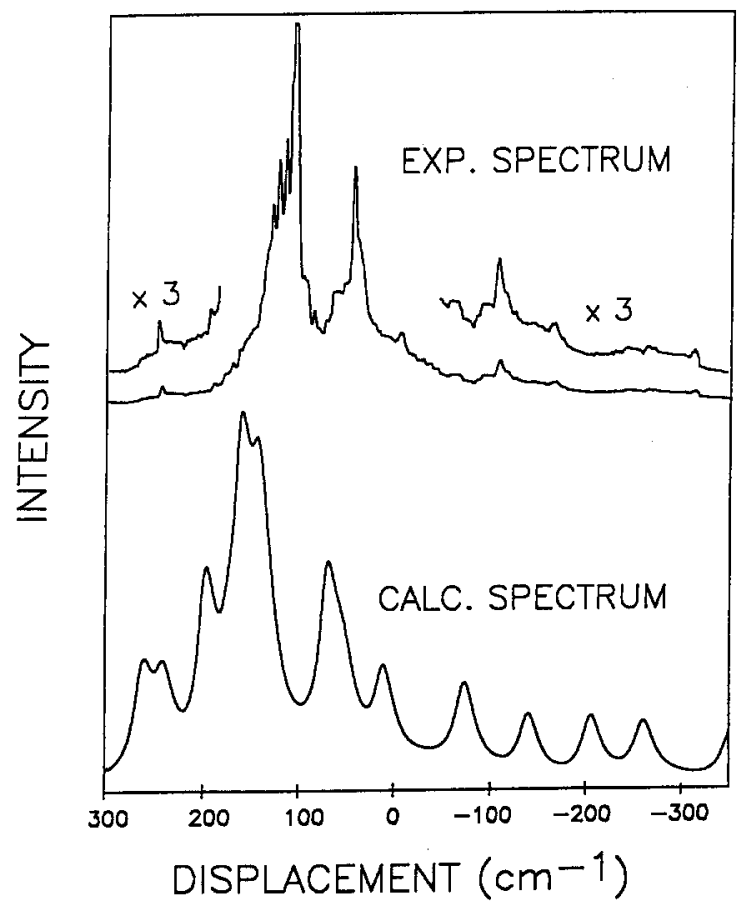

FIG. 8. The observed and calculated torsion-wagging $T_{1}-S_{0}$ spectra of thioacetaldehyde at $614 \mathrm{~nm}$. calculated to be displaced from the $0_{0}^{0} a_{1}$ electronic origin by $132.50 \mathrm{~cm}^{-1}$. Because of the clear definition of this band and its high calculated strength, 93.3, it is used as the starting point for the vibronic assignment. The second strongest band is observed at $16339.4 \mathrm{~cm}^{-1}$ and is assigned to $15_{0}^{1} e$. The observed interval between these two bands of $63.2 \mathrm{~cm}^{-1}$ compares favorably with the calculated interval of $68.80 \mathrm{~cm}^{-1}$. The perplexing part of the analyses is that the $15_{0}^{2} e$ band has a calculated relative

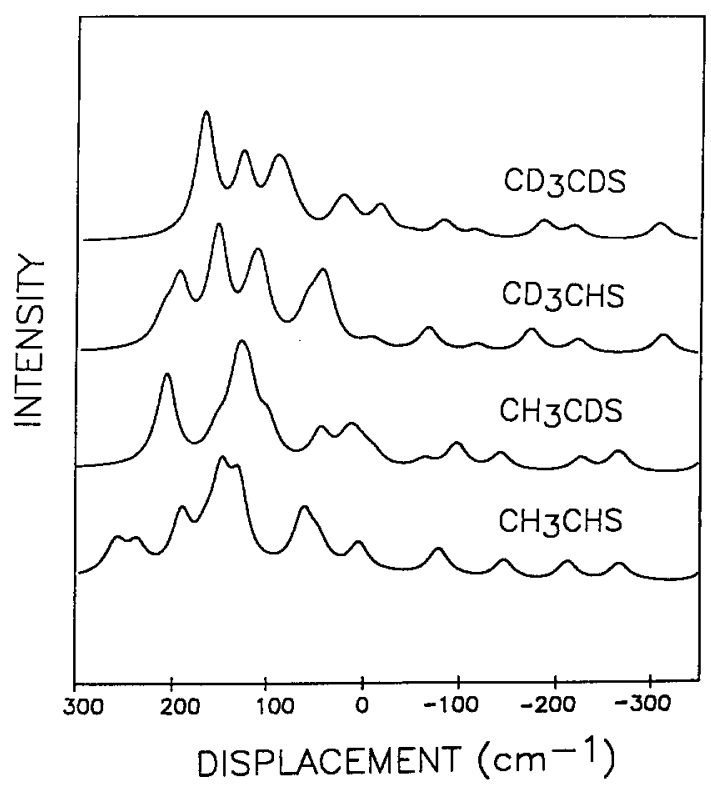

FIG. 9. The calculated spectra of thioacetaldehyde, $\mathrm{CH}_{3} \mathrm{CHS}, \mathrm{CH}_{3} \mathrm{CDS}$, $\mathrm{CD}_{3} \mathrm{CHS}$, and $\mathrm{CD}_{3} \mathrm{CDS}$. 
intensity of 100 and is not observed in the spectrum as a prominent feature. Instead, a broad absorption is observed at $+150 \mathrm{~cm}^{-1}$, which extends over more than $50 \mathrm{~cm}^{-1}$. It is this broad undulating feature that is assigned to $15_{0}^{2} e$. It would appear that the strongest band in the torsional progression has a contour that is different from that of the other bands in the spectrum in that it is broad and contains subband structure. This is somewhat surprising and a possible explanation for the different contour is that the upper level, $15^{2 e}$, lies just above the top of the central barrier. Without a hindering barrier, the methyl group would behave somewhat like a free rotor and the quantum numbers that describe the internal torsion would be those of the free internal rotor.

The origin band may not be observed directly in the absorption spectrum since it is calculated to have a strength of 10.0 and is adjacent to the stronger hot band $15_{1}^{2} e$ that is calculated to have an intensity of 27.4. The hot band structure at $16189.5,16131.4,16033.9$, and $15986.2 \mathrm{~cm}^{-1}$ is assigned without difficulty to the $15_{1}^{1} a_{2}, 15_{1}^{0} e, 15_{2}^{1} e$, and $15_{2}^{0} a_{1}$ vibronic transitions. When the origin is placed at $16292.8 \mathrm{~cm}^{-1}$, the levels of the $S_{0}$ state may be extracted by combination differences to give $15_{1 e} / 15_{2 a 1}$ of $161.4 / 306.6 \mathrm{~cm}^{-1}$ that may be directly compared to the calculated values of $144.68 / 264.85 \mathrm{~cm}^{-1}$. Thus, the $a b$ initio MO calculations underestimate the observed frequency data by about 11 percent. On the other hand, the $15^{1 e} / 15^{2 a 1}$ levels for the $T_{1}$ state are observed to be at $46.6 / 109.8 \mathrm{~cm}^{-1}$ and are some 37 percent lower than the calculated values, $63.73 / 132.50 \mathrm{~cm}^{-1}$. The observed band at $+249.6,16542.4 \mathrm{~cm}^{-1}$ cannot be assigned to transitions to higher quanta of the $Q_{15}$ mode. Rather it must arise from the activity of the aldehyde wagging mode. Such transitions would be forbidden in the absence of wagging-torsion coupling. In the calculated spectrum, the transition $14_{0}^{1} 15_{0}^{1} e$ at $+238.98 \mathrm{~cm}^{-1}$ has a calculated strength of 31.5. While the agreement between the calculated and observed line positions is good, the calculations overestimate the strength of this band.

Further work is planned on this system using the technique of flash pyrolysis-supersonic jet spectroscopy. The reduction in the vibrational temperature by this method is expected to remove most of the torsional hot band struc- ture and the background congestion and to give a better view of the higher vibronic bands in the spectrum. This information will be invaluable in establishing the band assignments and should give further insight into the anharmonic coupling between the two large amplitude low frequency modes.

${ }^{1}$ D. C. Moule and A. D. Walsh, Chem. Rev. 75, 67 (1975).

${ }^{2}$ D. J. Clouthier and D. A. Ramsay, Annu. Rev. Phys. Chem. 34, 13 (1983).

${ }^{3}$ J. S. Crighton and S. Bell, J. Mol. Spectrosc. 112, 315 (1985).

${ }^{4}$ M. Baba, V. Nagashima, and I. Hanazaki, J. Chem. Phys. 83, 3514 (1985).

${ }^{5}$ R. P. Steer, Rev. Chem. Intermed. 4, 1 (1981).

${ }^{6}$ S. Paone, D. C. Moule, A. E. Bruno, and R. P. Steer, J. Mol. Spectrosc. 107, 1 (1984).

${ }^{7}$ R. H. Judge, D. C. Moule, A. E. Bruno, and R. P. Steer, J. Chem. Phys. 87, 60 (1987).

${ }^{8}$ A. E. Bruno, D. C. Moule, and R. P. Steer, J. Photochem. 46, 169 (1989).

${ }^{9}$ H. W. Kroto and B. M. Landsberg, J. Mol. Spectrosc. 62, 346 (1976).

${ }^{10}$ Y. G. Smeyers and M. N. Bellido, Int. J. Quantum Chem. 19, 553 (1981).

${ }^{11}$ S. L. Altmann, Proc. R. Soc. Ser. A 298, 184 (1967).

${ }^{12}$ C. M. Woodman, Mol. Phys. 19, 753 (1970).

${ }^{13}$ H. C. Longuet-Higgins, Mol. Phys. 6, 445 (1963).

${ }^{14}$ J. Maruani, A. Hernandez-Laguna, and Y. G. Smeyers, J. Chem. Phys. 63, 4515 (1975).

${ }^{15}$ C. R. Quade and C. L. Lin, J. Chem. Phys. 38, 540 (1963); C. R. Quade, ibid, 47, 1073 (1967); S. V. Knopp and C. R. Quade, ibid. 48, 3317 (1968).

${ }^{16}$ Y. G. Smeyers and A. Hernandez-Laguna, Int. J. Quantum Chem. 22, 68 (1982); J. Mol. Struct. (Theochem.) 149, 127 (1987).

${ }^{17}$ J. Maruani, Y. G. Smeyers, and A. Hernandez-Laguna, J. Chem. Phys. 76, 3123 (1982).

${ }^{18}$ Y. G. Smeyers, J. Mol. Struct. (Theochem.) 107, 3 (1984).

${ }^{19}$ Y. G. Smeyers, M. N. Bellido, and A. Nino, J. Mol. Struct. (Theochem.) 166, 1 (1988).

${ }^{20}$ Y. G. Smeyers, A. Nino, M. N. Bellido, Theoret. Chim. Acta. 74, 259 (1988).

${ }^{21}$ J. E. Kilpatrick and K. S. Pitzer, J. Chem. Phys. 171064 (1949).

${ }^{22}$ E. B. Wilson, Jr., J. Chem. Phys. 3, 276 (1935).

${ }^{23} \mathrm{~J}$. M. Peterson and R. Poirier, University of Toronto, 1980.

${ }^{24}$ J. D. Lewis, T. B. Malloy, T. H. Chao, and J. Laane, J. Mol. Struct. 12, 427 (1972).

${ }^{25}$ F. Ioannoni, D. C. Moule, J. D. Goddard, and D. J. Goddard, J. Mol. Struct. 197, 159 (1989).

${ }^{26}$ K. B. Wiberg, V. Waltersand, and S. D. Colson, J. Phys. Chem. 88, 4723 (1984).

${ }^{27}$ I. C. Hisatsune and J. Heicklen, Can. J. Spectrosc. 18, 135 (1973).

${ }^{28}$ DI-3000. Copyright 1984 by Precision Visuals, Inc. 